\title{
Elektronische Zeitung - das Erbe Gutenbergs?
}

Die Digitalisierung im Medienumfeld ist bereits seit vielen Jahren allgegenwärtig und schreitet weiter voran: während die $C D$ und DVD in viele Alltagsbereiche bereits Einzug gehalten haben, verbreiten sich koexistent zu ihren analogen Geschwistern vor allem das digitale Fernsehen, mit Startschwierigkeiten auch das digitale Radio (Digital Audio Broadcasting). Ergänzt wird das Angebot elektronischer Informationsmedien durch das Internet, die Möglichkeit der digitalen Telefonübertragung und die Verwendbarkeit elektronischer Organizer (Handhelds bzw. PDAs). Zwei technologieübergreifende Phänomene lassen sich in diesem Kontext identifizieren: (1) Das vermehrte Zusammenspiel der Technologien (Technologiekonvergenz); als prominente Beispiele seien die Internettelefonie und das interaktive Fernsehen genannt. (2) Die Trennung zwischen Inhalt und seiner Darstellungsform bzw. des inn illustrierenden Mediums; so lässt sich dieselbe E-Mail mit einem Internet-Client, auf dem Mobiltelefon oder dem Handheld medienadäquat darstellen.

Im Printmedienbereich nehmen die digitalen Technologien mit Ausnahme der Druckvorstufe weiterhin nur eine Randposition ein. Es existieren zwar für zahlreiche Zeitungen und Zeitschriften

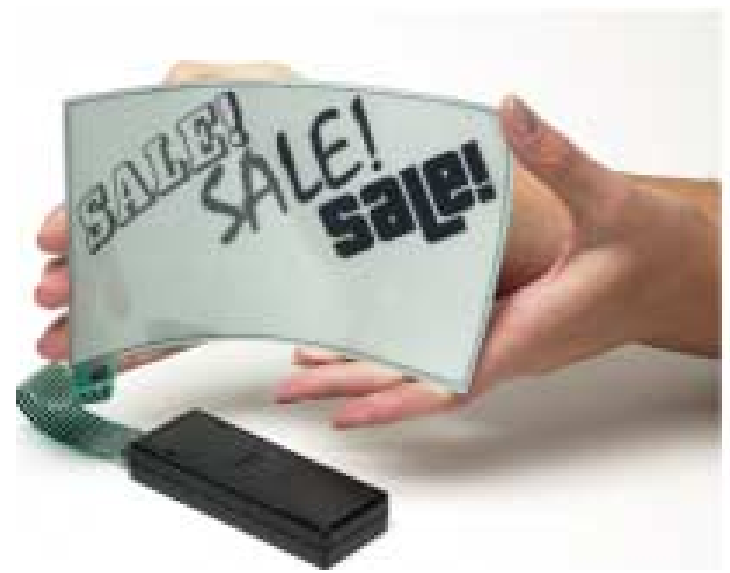

Bild 1: Elektronisches Papier und Verwendung als Werbeplakat, Quellen: E Ink 2002, Gyricon 2002

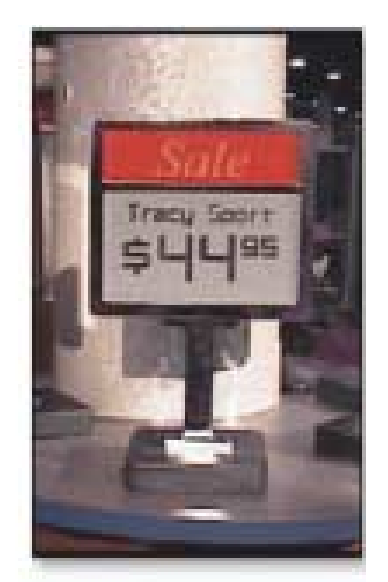

Webseiten, diese haben jedoch in der Regel keinen Substitutcharakter: Sie enthalten meistens nur Auszüge aus den Printausgaben. Auch scheint ihr Substitutpotential gering, da die Attraktivität von Zeitungen und Zeitschriften vor allem in ihrer flexiblen Handhabbarkeit im Alltag zu sehen ist: Sie lassen sich falten, platzsparend transportieren und in der U-Bahn und dem Flugzeug ebenso lesen wie in der Kantine und am Strand.

Einen neuen Weg weist die elektronische Zeitung bzw. das elektronische Papier, das aus einem flexiblen und biegsamen Material besteht. Der Terminus "elektronische Zeitung" findet sich in der Literatur unter verschiedenen Begriffsverständnissen diskutiert: In diesem Artikel wird nicht die Online- bzw. Internetausgabe einer Zeitung thematisiert, sondern ein auf einer materialtechnologischen Entwicklung des elektronischen Papiers basierendes Papiersubstitut, das die ergonomische Flexibilität bzw. Faltbarkeit des Papiers mit einer digitalen Wiederbeschreibbarkeit vereint (eNewspaper oder electronic newspaper). Bild 1 illustriert eine derartige Folie, deren breites Anwendungsspektrum neben der elektronischen Zeitung auch Werbeplakate oder Monitordisplays ${ }^{1}$ umfasst.
Die Entwicklung adäquater Materialien und Technologien zur Realisierung eines Papiersubstitutes, das die elektronische Darstellung von Inhalten erlaubt, liegt derzeit in US-oligopoler Hand² (Angerer 2001, Ditlea 2001, Kunkel 2000 und Siemens 1999). Eine Verknüpfung von Forschung und Produktentwicklung wird von den Mitbewerbern E Ink und Gyricon Media vorgenommen, die aus dem Massachusetts Institut of Technology (MIT) bzw. aus dem Xerox Palo Alto Research Center hervorgegangen sind. ${ }^{3}$

Die Technologien von E Ink und Gyricon basieren auf mikroskopisch kleinen, etwa haardünnen Kugeln, die in Abhängigkeit von der durch Elektroden erzeugten Ladung eine helle oder dunkle Ausprägung annehmen und in transparente Spezialfolien eingebettet sind. Die einzelnen Darstellungseinheiten bzw. ihre Elektroden lassen sich über einen Displaycontroller ansprechen. Während Gyricon für eine Darstellungseinheit eine Kugel verwendet, finden sich bei $\mathrm{E}$ Ink jeweils mehrere (siehe Bild 2). Detaillierte Informationen finden sich auf den Webseiten der Unternehmen ( $E$ Ink 2002 und Gyricon 2002) sowie bei Ditlea 2001 und Ditlea 2002. Forschungsarbeit wird u.a. noch im Bereich einer adäquaten Auflösung geleistet; E Ink arbeitet in Kooperation mit dem japani-

\footnotetext{
3 Zur Historie des E-Papers und der beteiligten
Forschungsinstitute sei auf Jacobson et al. 1997, Kunkel 2000 und Peterson 1998 verwiesen.

${ }^{1}$ Die Unternehmen E-Ink und Philips sind 2001 eine Kooperation zur Nutzung des elektronischen Papiers für Displays eingegangen (s. Pressemeldungen wie bspw. E Ink 2001).

2 Dies gilt nicht für den Bereich der „Organischen Licht Emittierenden Dioden" (OLED), in dem beispielsweise Siemens forscht (Siemens 1999), der aber derzeit für elektronische Zeitungsdisplays keine bedeutende Rolle einnimmt.
} 


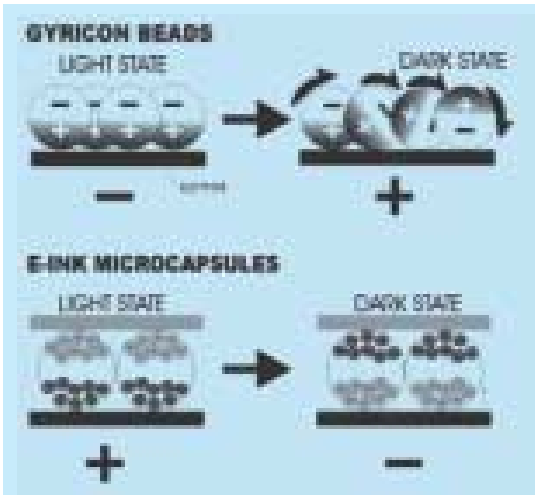

Bild 2: Funktionsweise von E-Paper der Unternehmen Gyricon und E Ink, Quelle: Ditlea 2001

schen Unternehmen Toppan Printing an einer Farbversion des E-Papers (E Ink 2002, Printprocess 2001). Eine detailliertere Behandlung der materialtechnologischen Facette sowie eine Beleuchtung der Einsatzpotentiale über die elektronische Zeitung hinaus findet sich bei Schryen und Karla 2002.

Derzeitig prototypische Einsätze der Technologien finden sich bei Anzeigetafeln in Kaufhäusern und bei Nachrichten-Displays von Zeitungsverlagen (Ditlea 2001), jedoch noch nicht im Rahmen von elektronischen Zeitungen. Von der konkreten Realisierung kann hingegen im Rahmen der weiteren Betrachtungen abstrahiert werden; Es wird von einem fiktiven Ausgabegerät ausgegangen. Bzgl. des von IBM 1999 vorgestellten und in der Literatur oft aufgegriffenen Designkonzepts (Deider, Fuhlrott 1999 und IDSA 1999) sei darauf hingewiesen, dass es sich ausschließlich um ein nichtfunktionales Rahmendesign handelte, in das Papierseiten eingelegt wurden (Steinbugler 2001).

Die elektronische Zeitung wirft aufgrund ihres gesamtgesellschaftlichen Charakters Fragestellungen in diversen (wissenschaftlichen) Disziplinen auf, die bislang wohl aufgrund ihrer noch visionären Eigenschaft kaum adressiert wurden. Soziologische, ökologische, rechtswissenschaftliche sowie volkswirtschaft- liche Fragestellungen koexistieren mit technologischen und betriebswirtschaftlichen Aufgaben. Während eine materialtechnologische Reifung des elektronischen Papiers hinsichtlich Auflösung, Farbdarstellung und Robustheit sowie erste Produktdefinitionen im Bereich von Displays zu beobachten sind - das Design einer ergonomisch sinnvollen und erfolgreichen elektronischen Zeitung als Produkt ist indes noch unklar -, stellen sich im wirtschaftswissenschaftlichen und -informatischen Bereich u.a. die Fragen, wie hoch die Marktakzeptanz seitens der Verlage und Leser ist, welche Gestalt informations- und kommunikationstechnologische Landschaften zur Unterstützung der veränderten Wertschöpfungskette und Geschäftsmodelle haben, welche Migrationsstrategien sich für die Verlage anbieten und wie sich die elektronische Zeitung in die digitale Medienlandschaft integrieren lässt. Eine der letzten Domänen der nicht-digitalen Medienwelt wird vermutlich nicht innerhalb der nächsten Monate und Jahre neu erobert werden, aber die Möglichkeit der Integration der elektronischen Zeitung in die digitale Medienwelt und damit auch die Notwendigkeit ihrer wirtschaftsinformatischen Adressierung sind vorgezeichnet.

\section{Literatur}

Angerer, S.: Aktuell in Ewigkeit. W\&V 40 (2001) 182-183.

Deider, C.; Fuhlrott, R.: Elektronische Zeitung von IBM. B.I.T. online 04 (1999), Digit@| News. http://www.b-i-t-online.de/archiv/1999-04/digit1.htm (Letzter Zugriff: 18.10.2002).

Ditlea, S.: The Electronic Paper Chase. American Scientific Nov. (2001). http://www.sciam.com/2001/1101issue/1101ditlea. html (Letzter Zugriff: 19.05.2002).

Ditlea, S.: Wettlauf zum elektronischen Papier. Spektrum der Wissenschaft 05 (2002) 4651.

E Ink: Pressemitteilung vom 27.02.2001: PHILIPS INVESTS IN E INK, SECURES GLOBAL COMMERCIAL RIGHTS FOR USE OF ELECTRONIC
INK IN HANDHELD DISPLAYS. http://eink com/news/releases/pr28.html (Letzter Zugriff: 18.10.2002).

E Ink: Webseite von E Ink: http://www eink.com (Letzter Zugriff: 18.10.2002).

Gyricon: Webseite von Gyricon Media: http:// www.gyriconmedia.com/smartpaper/ index.asp (Letzter Zugriff: 18.10.2002).

IDSA (Industrial Designers Society of America): IBM Electronic Newspaper. http://wwww. idsa.org/whatis/seewhat/idea99/winners/epaper.htm (Letzter Zugriff: 18.10.2002).

Jacobson, J.; Comiskey, B.; Turner, C.;Albert, J.;Tsao, P.: The last book. IBM Systems Journal 3 (1997) 457-463.

Kunkel, P.: Scrap the presses - print and the Web are racing toward the biggest media merger in history. Wired Aug. (2000). http:// www.wired.com/wired/archive/8.08/ epapers.html (Letzter Zugriff: 18.10. 2002).

Peterson, I.: Rethinking Ink: Printing the pages of an electronic book. Science News Online $\mathbf{2 5}$ (1998) 396ff.

Printprocess: Urlaubsschmöker auf E-PAPIER eingewebt im Badetuch. print process $\mathbf{1 5}$ (2001) 13.

Schryen, G.; Karla, J.: Elektronisches Papier - Displaytechnologie mit weitem Anwendungsspektrum. Wirtschaftsinformatik (erscheint in Kürze).

Siemens: Leuchtende Kunststoffe: Flach, flexibel, futuristisch - die organischen Leuchtdioden. Forschung und Innovation 2 (1999). http://w4.siemens.de/Ful/de/archiv/zeitschrift/heft2_99/artikel08/index.html (Letzter Zugriff: 18.10.2002).

Steinbugler: Persönliche Email des verantwortlichen Designstudienleiters von IBM Robert Steinbugler im Herbst 2001

Wirtz, B. W.: Medien- und Internetmanagement. 2. Auflage. Wiesbaden: Gabler, 2001

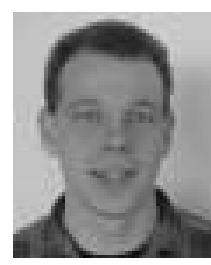

Dr. Guido Schryen, M.O.R., Lehrstuhl für Wirtschaftsinformatik und Operations Research der RWTH Aachen, Hauptarbeitsgebiete: Elektronische Medien, E-Business.

E-Mail: schryen@winfor.rwth-aachen.de 